\title{
5/6 Nephrectomy as a Validated Rat Model Mimicking Human Warfarin-Related Nephropathy
}

\author{
A. Ozcan ${ }^{a}$ d $\quad$ K. Ware ${ }^{a} \quad$ E. Calomeni ${ }^{a} \quad$ T. Nadasdy $^{a} \quad$ R. Forbes ${ }^{c}$ A.A. Satoskar ${ }^{a}$ \\ G. Nadasdy $^{a} \quad$ B.H. Rovin ${ }^{b} \quad$ L.A. Hebert ${ }^{b} \quad$ S.V. Brodsky ${ }^{a}$
}

Departments of a Pathology and ${ }^{b}$ Medicine, ${ }^{c}$ Comprehensive Transplant Center, The Ohio State University, Columbus, Ohio, USA; d Department of Pathology, Gulhane Military Medical Academy, Ankara, Turkey

\section{Key Words \\ Warfarin-related nephropathy · Acute kidney injury • \\ Coagulation $\cdot 5 / 6$ Nephrectomy}

\begin{abstract}
Background/Aims: We previously reported that patients with chronic kidney disease (CKD) receiving warfarin therapy and whose international normalized ratio increases to $>3.0$ may develop acute kidney injury (AKI) as a result of glomerular hemorrhage and formation of obstructive red blood cell (RBC) casts. We named this condition warfarin-related nephropathy (WRN). We also previously reported that acute excessive anticoagulation with brodifacoum (superwarfarin) induces $A K I$ in $5 / 6$ nephrectomy (5/6NE) rats. Limitations of the brodifacoum model precluded a careful assessment of dose-response relationships. Methods: Warfarin treatment was used in 5/6NE. Results: Herein we report that warfarin treatment of 5/6NE rats resulted in a dose-dependent increase in serum creatinine (SC). The increase in SC following warfarin treatment was greater at 3 and 19 weeks after the ablative surgery, than that observed 8 weeks after the ablative surgery. The SC increase was correlated with the prothrombin time increase. Morphologically, 5/6NE, but not control rats, had acute tubular injury with $\mathrm{RBC}$ and $\mathrm{RBC}$ casts
\end{abstract}

in the tubules. Treatment with vitamin $\mathrm{K}$ prevented SC increase and morphologic changes in the kidney associated with warfarin treatment. A single episode of WRN did not affect the progression of CKD in 5/6NE. Conclusion: (1) The $5 / 6 \mathrm{NE}$ model of CKD is an appropriate animal model to study the pathogenesis of WRN. (2) The pharmacokinetics of warfarin is better suited to the study of WRN than that of brodifacoum. (3) The more advanced stages of 5/6NE are more susceptible to WRN than the earlier stages. (4) Vitamin K treatment prevents WRN.

Copyright $\odot 2012$ S. Karger AG, Basel

Warfarin-related nephropathy (WRN) is a newly recognized entity that we recently described. WRN is the result of excessive anticoagulation with warfarin (international normalized ratio [INR] $>3.0$ ) which results in glomerular hemorrhage, occlusive tubular red blood cell (RBC) cast formation [1] and acute kidney injury (AKI) in susceptible patients $[2,3]$. WRN is not an uncommon complication of warfarin therapy, occurring in about $16 \%$ of non-chronic kidney disease (CKD) patients [2] and $37 \%$ of CKD patients $[2,3]$ on warfarin treatment who had at least one episode of increased INR $>3.0$. Progression of CKD is accelerated by WRN [2]. Also, WRN is

\section{KARGER}

Fax +41613061234

E-Mail karger@karger.ch

www.karger.com
(C) 2012 S. Karger AG, Basel

0250-8095/12/0354-0356\$38.00/0

Accessible online at:

www.karger.com/ajn
Assist. Prof. Sergey V. Brodsky, MD, PhD

Department of Pathology, The Ohio State University

333 W 10th Ave, Graves Hall, B078

Columbus, $\mathrm{OH} 43210$ (USA)

Tel. +1 614688 5831, E-Mail sergey.brodsky@osumc.edu 
associated with increased mortality [3] and some patients lost their kidney function permanently and required dialysis [5].

The need to elucidate WRN pathogenesis is emerging because without an understanding of WRN pathogenesis it would be difficult to develop pharmacologic strategies for WRN prevention and treatment. Recently we reported that excessive anticoagulation in 5/6 nephrectomy (5/6NE) rats by brodifacoum (superwarfarin) results in $\mathrm{AKI}$ and morphologic findings in the kidneys comparable to those observed in patients with WRN [4].

A limitation of the brodifacoum model of $\mathrm{WRN}$ is that it results in a rapid increase in prothrombin time (PT), which precludes a careful assessment of dose-response relationships between coagulopathy and serum creatinine (SC) changes. Also, the brodifacoum model does not reproduce the clinical scenario of WRN in patients in which the AKI appears to evolve more gradually $[2,3]$.

The aims of this study were to investigate the pathogenesis of WRN using warfarin in the 5/6NE of CKD. The key aims to explore are: (1) the association between the degree of anticoagulation and renal function; (2) the possibility to prevent or treat WRN by vitamin K administration; (3) the role of a single episode of WRN on the progression of CKD.

\section{Concise Methods}

All experiments were conducted in accordance with the NIH Guide for the Care and Use of Laboratory Animals [5]. The 5/6NE was performed in male Sprague Dawley rats by a nephrectomy of the right kidney and resection of two thirds of the left kidney, as previously described [4]. Warfarin was given per os in drinking water. The daily amount of water consumption was measured and the warfarin dose was calculated based on the animal weight. Vitamin $\mathrm{K}$ was administered by an intraperitoneal injection.

SC was measured using a Creatinine Reagent Assay (Raichem, San Marcos, Calif., USA) according to the manufacturer protocol. PT was measured using Electra 750 coagulation analyzer (Medical Laboratory Automation, Pleasantville, N.Y., USA) according to the manufacturer protocol. Hematuria and proteinuria were measured using DiaScreen (Chronimed Inc., Minnetonka, Minn., USA) reagent strips in the urine. For kidney morphology, hematoxylin and eosin-stained $3-\mu \mathrm{m}$ sections of paraffin-embedded kidneys were analyzed. In each control animal, the entire area of longitudinal sections of one kidney was evaluated. In 5/6NE, the scarred areas related to the surgical procedure were excluded.

\section{Statistical Analysis}

Results are presented as mean \pm standard error if not otherwise specified. Differences between groups were analyzed by the two-paired $t$ test or ANOVA test, where it was applicable. Tukey post-test was performed to analyze the differences between groups in conjunction with ANOVA. Association between SC changes and PT increase was analyzed using Pearson correlation analysis with two-tailed $p$ value.

\section{Results}

Warfarin Treatment Results in a Gradual PT Increase in Experimental Animals

Warfarin was given in drinking water in a dose of 0.20 $\mathrm{mg} / \mathrm{kg} / 24 \mathrm{~h}$ for the first week of treatment, $0.34 \mathrm{mg} / \mathrm{kg} /$ $24 \mathrm{~h}$ for the second week of treatment and $0.75 \mathrm{mg} / \mathrm{kg} /$ $24 \mathrm{~h}$ for the third week of treatment. This regimen resulted in a gradual increase in PT in all experimental groups (fig. 1).

We used a 'surrogate' INR (sINR) by comparing PT after and before the treatment, as it was described earlier [4].

Warfarin treatment did not affect either systolic or diastolic blood pressure in 5/6NE or control rats (data not shown).

\section{PT Increase Is Accompanied by Progressive}

Hematuria in Experimental Animals.

As we had described earlier, 5/6NE results in progressive hematuria [4]. Hematuria was significantly increased in 5/6NE animals treated with warfarin. Animals treated with warfarin at 8 or 19 weeks after ablative surgery $(8 \mathrm{~W}$ and $19 \mathrm{~W}$ ) had progressive hematuria as their sINR was increasing. Interestingly, animals treated at $3 \mathrm{~W}$ had a surge in hematuria early in the treatment, when sINR was still in the therapeutic range. Later, the increase in hematuria in those animals was similar to hematuria changes in animals treated at $8 \mathrm{~W}$ or $19 \mathrm{~W}$. Hematuria also increased in control animals treated with warfarin, but the degree of hematuria was significantly less as compared to $5 / 6 \mathrm{NE}$, and the hematuria increased only when sINR increased above $2 \mathrm{AU}$ (fig. 1).

\section{Increase in PT Is Associated with Increase in SC}

SC was significantly increased in 5/6NE rats treated with warfarin as compared to the vehicle-treated rats with the same progression of chronic kidney injury. In control rats (without the ablative surgery), warfarin treatment resulted only in fluctuations in SC levels without a significant trend (fig. 2).

Next, we analyzed if changes in SC levels were associated with PT changes. In all experimental groups changes in SC levels were correlated with PT changes (mea- 


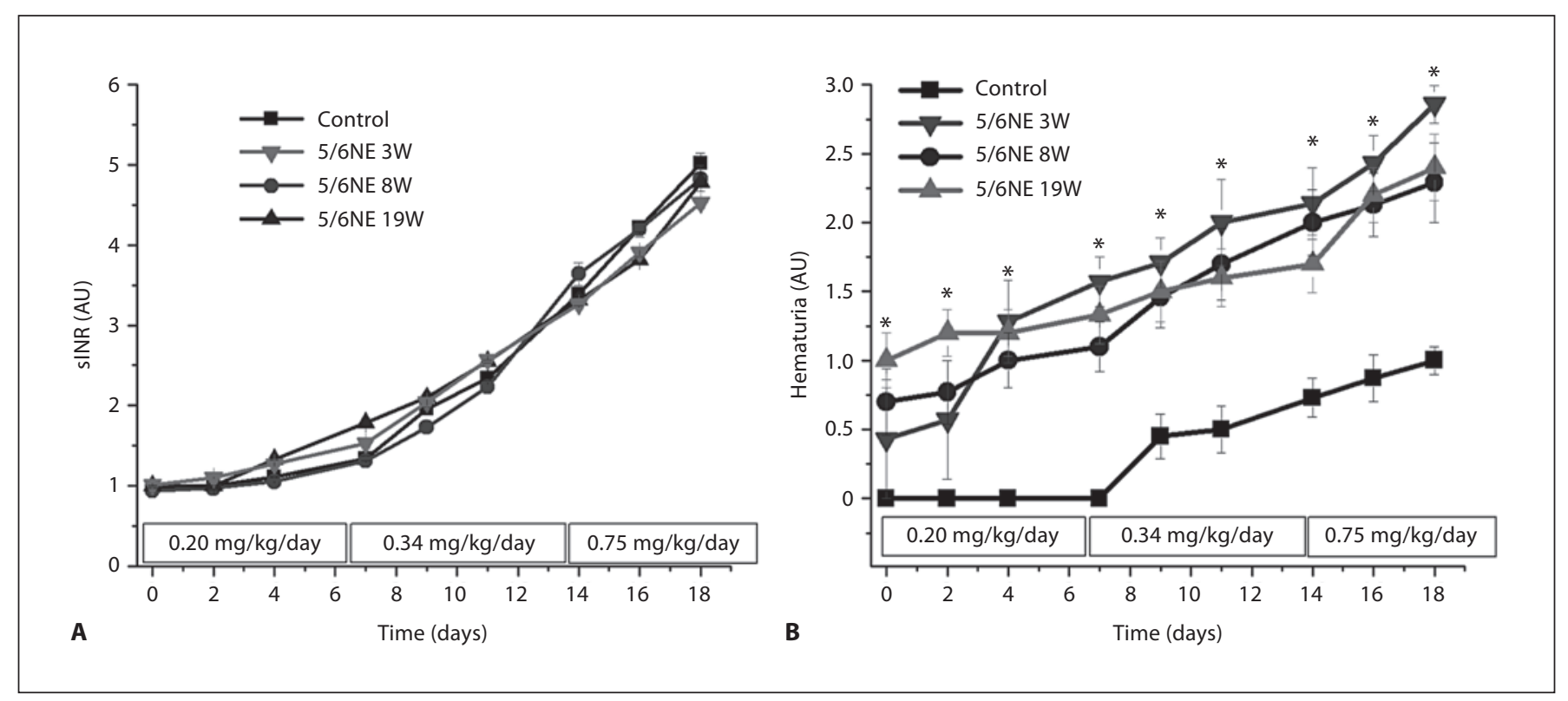

Fig. 1. Changes in prothrombin time (PT) and hematuria in animals treated with warfarin. A Warfarin was administered per os (doses and time are shown in rectangles on the $\mathrm{x}$-axis). This protocol resulted in a gradual increase in PT (sINR is shown) in all experimental groups. B Hematuria was measured using DiaScreen (Chronimed Inc., Minnetonka, Minn., USA) reagent strips in control (solid squares, $\mathrm{n}=11$ ) and $5 / 6$ nephrectomy rats at 3 weeks $(5 / 6 \mathrm{NE} 3 \mathrm{~W}$, inverted triangles, $\mathrm{n}=7)$, 8 weeks $(5 / 6 \mathrm{NE} 8 \mathrm{~W}$, solid circles, $\mathrm{n}=10)$ and 19 weeks $(5 / 6 \mathrm{NE} 19 \mathrm{~W}$, upright triangles, $\mathrm{n}=6$ ) after the ablative surgery. Hematuria was graded using a semiquantitative scale of $0-3+$. Score 0 was designated for negative hematuria, score $1+$ for mild hematuria, score $2+$ for moderate hematuria and score $3+$ for large hematuria. ${ }^{*} \mathrm{p}<0.05 \mathrm{com}-$ pared to control. sured as sINR). The lowest correlation coefficient $\left(\mathrm{R}^{2}\right.$ value) was in the control group $\left(\mathrm{R}^{2}=0.054, \mathrm{p}=0.0131\right)$. Although statistically significant, this correlation was much weaker as compared to 5/6NE. In 5/6NE, the strongest correlation between SC and sINR was found in 5/6NE rats treated with warfarin at $3 \mathrm{~W}$ or $19 \mathrm{~W}\left(\mathrm{R}^{2}=0.570, \mathrm{p}<\right.$ 0.0001 and $\mathrm{R}^{2}=0.67, \mathrm{p}<0.0001$, respectively), whereas in $5 / 6 \mathrm{NE}$ rats treated with warfarin at $8 \mathrm{~W}$ this association was weaker $\left(\mathrm{R}^{2}=0.310, \mathrm{p}<0.0001\right)$, but still stronger as compared to control animals.

\section{Warfarin Treatment Results in Kidney Morphology} Changes Comparable to Those Seen in Humans with WRN.

Pathologic findings in the kidneys obtained from animals treated with warfarin are depicted in table 1 . In $5 / 6 \mathrm{NE}$ rats treated with warfarin at $8 \mathrm{~W}$, increase in PT was associated with an increased number of tubules containing RBC and $\mathrm{RBC}$ casts in both renal cortex and renal medulla. Occasional glomeruli had RBC in the Bowman's space, similar to our previous observation in animals treated with brodifacoum (fig. 3). Acute tubular in- jury increased at higher PT. These findings are similar to those identified in kidney biopsies from patients with WRN [1] and 5/6NE rats treated with brodifacoum [4]. Chronic kidney injury at different stages of CKD progression was similar in all animals with different PT values.

Treatment with Vitamin K Prevents Warfarin Effects on SC Levels and Kidney Morphology

To investigate whether the AKI associated with excessive anticoagulation with warfarin can be prevented by vitamin $\mathrm{K}$, animals were treated with a high warfarin dose $(0.75 \mathrm{mg} / \mathrm{kg} / 24 \mathrm{~h})$ and vitamin $\mathrm{K}(40 \mathrm{mg} / \mathrm{kg} / 24 \mathrm{~h})$ or vehicle. Warfarin-treated animals receiving vehicle had a rapid increase in $\mathrm{PT}$, whereas animals co-treated with warfarin and vitamin $\mathrm{K}$ did not develop significant $\mathrm{PT}$ increase (fig. 4).

Treatment with vitamin $\mathrm{K}$ prevented warfarin-associated increase in SC in 5/6NE animals treated at $3 \mathrm{~W}$ and $8 \mathrm{~W}$. In control animals no significant changes in SC were observed in animals treated either with the high dose of warfarin alone or together with vitamin K (fig. 3). Co- 


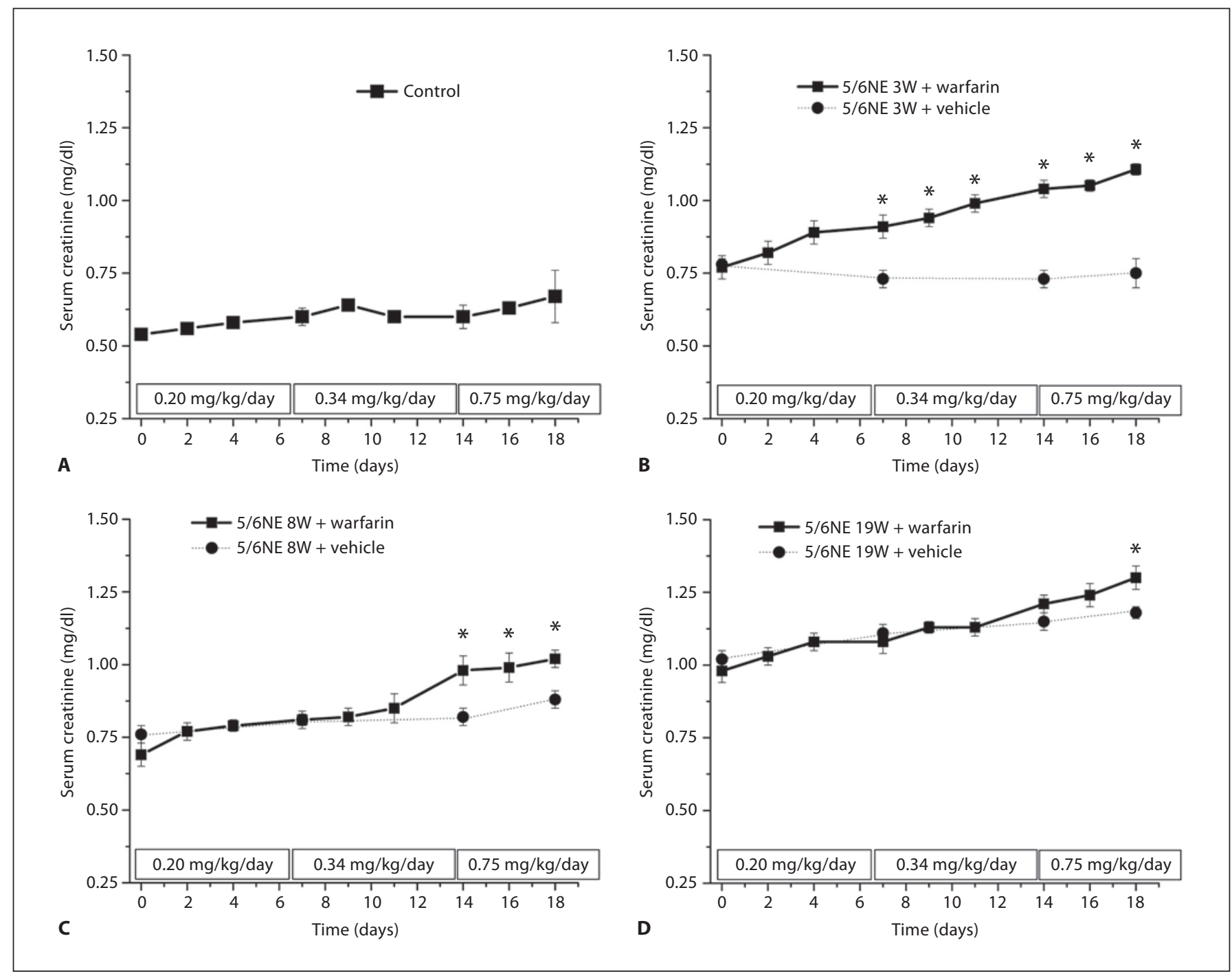

Fig. 2. Changes in serum creatinine (SC) levels in animals treated with warfarin. A Control (solid squares, $\mathrm{n}=11$ ), B 5/6 nephrectomy rats 3 weeks after the ablative surgery $(5 / 6 \mathrm{NE} 3 \mathrm{~W}, \mathrm{n}=7), \mathbf{C} 5 / 6$ nephrectomy rats 8 weeks after the ablative surgery $(5 / 6 \mathrm{NE} 8 \mathrm{~W}, \mathrm{n}=$ 10) and D 5/6 nephrectomy rats 19 weeks after the ablative surgery $(5 / 6 \mathrm{NE} 19 \mathrm{~W}, \mathrm{n}=6)$ were treated with increasing doses of warfarin

(doses and time are shown in rectangles on the $\mathrm{x}$-axis). Changes in SC levels in animals treated with warfarin (+ warfarin) (solid line, solid squares, A-D) were compared with SC levels in animals treated with vehicle (+ vehicle) (dotted line, solid circles, B-D) at the same time after the ablative surgery as the warfarin-treated animals. ${ }^{*} \mathrm{p}<0.05$ compared to vehicle-treated animals.

treatment with warfarin and vitamin K prevented formation of tubular RBC casts and acute tubular injury in 5/6NE animals at both $3 \mathrm{~W}$ and $8 \mathrm{~W}$ (table 1).

\section{A Single Episode of WRN Does Not Affect the}

Progression of Chronic Kidney Injury in 5/6NE Rats.

To investigate whether a single episode of WRN affects the progression of chronic kidney disease, as we observed in patients with WRN [3], 5/6NE rats were treated per os

with a high dose of warfarin $(0.75 \mathrm{mg} / \mathrm{kg} / 24 \mathrm{~h})$ until sINR achieved $>4.0$. This level of anticoagulation was achieved usually by the day 7-8 of treatment. At that time, warfarin treatment was stopped and the animals received intraperitoneal injection of vitamin $\mathrm{K}(40 \mathrm{mg} / \mathrm{kg})$. This resulted in a decrease of sINR to levels below $2 \mathrm{AU}$ within two days. SC was elevating during the warfarin treatment simultaneously with the increase in sINR, but SC rapidly decreased to the pretreatment values within two days af- 
Fig. 3. Morphologic findings in the kidneys obtained from 5/6 nephrectomy animals treated with warfarin. A Occasional glomeruli in 5/6 nephrectomy rats treated with warfarin had red blood cells (RBC) in the Bowman's space (arrow). The tubules associated with those glomeruli had RBC. B Numerous RBC occlusive casts were present in the tubules. Magnification $200 \times$. Hematoxylin-eosin stain.
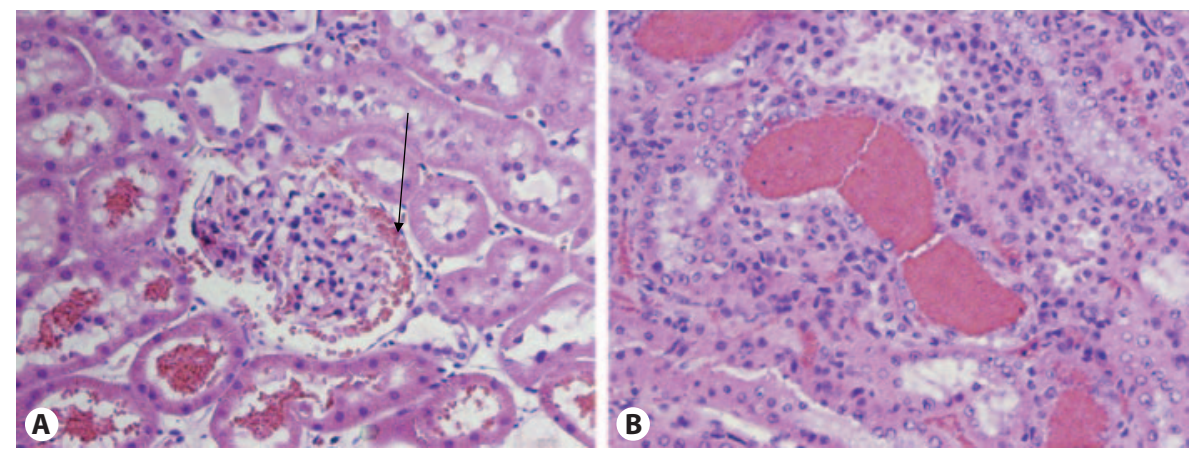

Table 1. Morphologic findings in control and 5/6 nephrectomy rats treated with warfarin

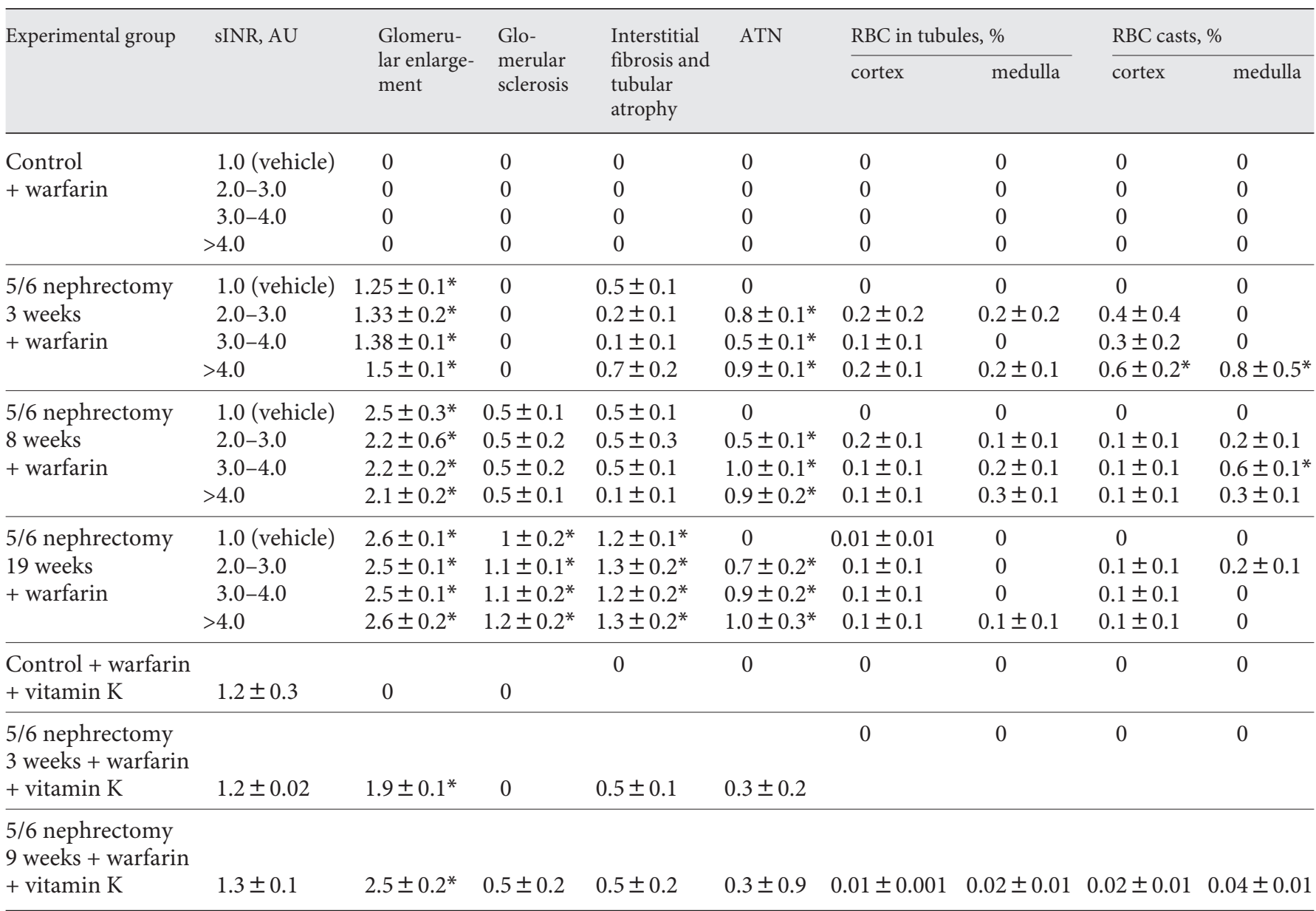

${ }^{*} \mathrm{p}<0.05$ compared to control.

Histological findings were scored semiquantitatively for each morphologic change assessed, mostly following the Banff criteria for allograft rejection. 

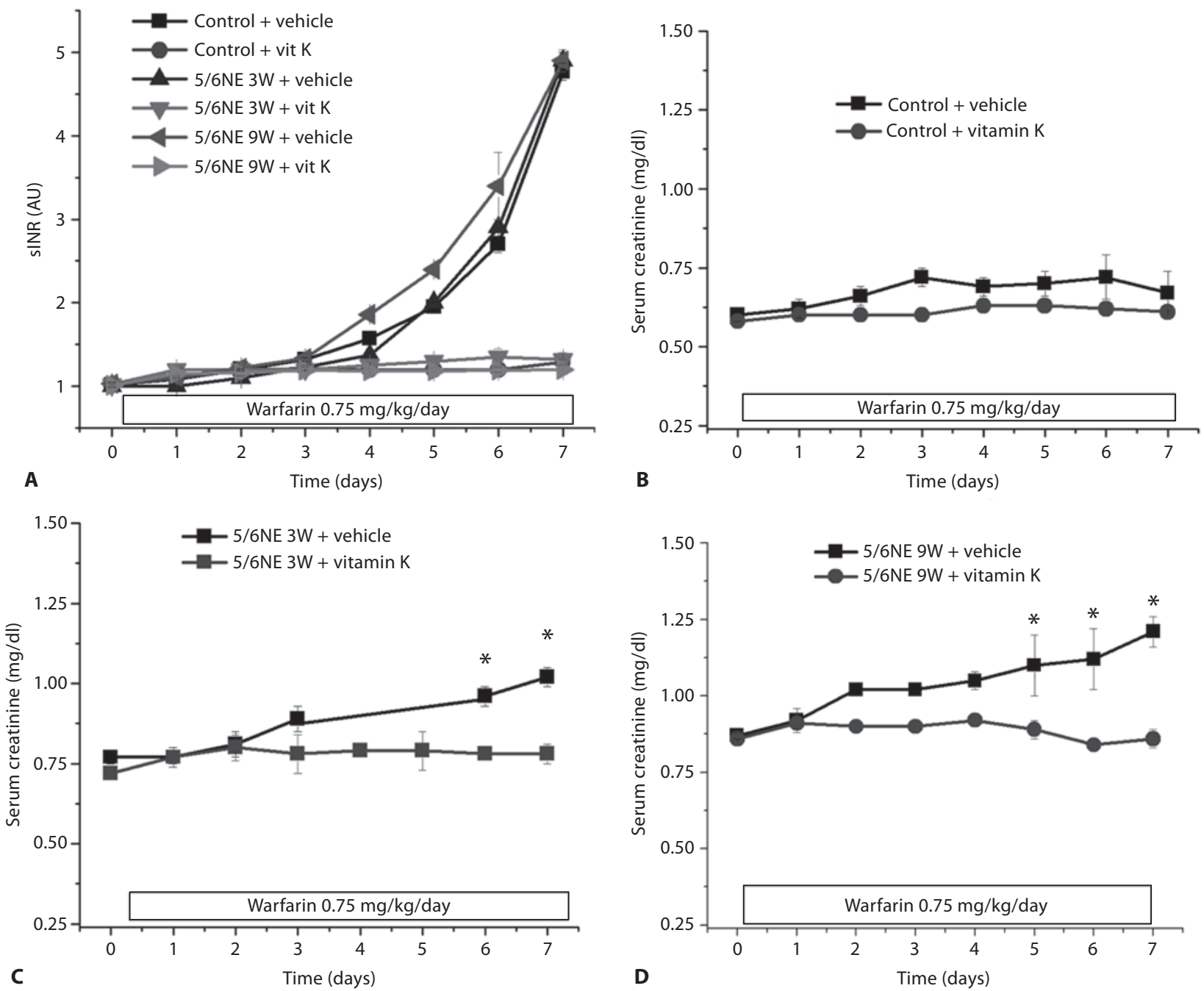

Fig. 4. Changes in serum creatinine (SC) levels in animals treated with warfarin and vitamin $\mathrm{K}$. Warfarin $(0.75 \mathrm{mg} / \mathrm{kg} / 24 \mathrm{~h})$ results in increased sINR above 4 within 1 week. Co-treatment with warfarin and vitamin $\mathrm{K}$ (vit K) $(40 \mathrm{mg} / \mathrm{kg} / 24 \mathrm{~h})$ prevented sINR increase (A). SC was increased in 5/6 nephrectomy rats treated with warfarin only, but not with warfarin and vitamin $\mathrm{K}$ together (B). A Control and 5/6 nephrectomy animals 3 weeks (5/6NE $3 \mathrm{~W}$ ) and 9 weeks $(5 / 6 \mathrm{NE} 9 \mathrm{~W})$ after the ablative surgery were treated with warfarin per os $(0.75 \mathrm{mg} / \mathrm{kg})$ and either vehicle (+ vehicle) or vitamin K (intraperitoneally $40 \mathrm{mg} / \mathrm{kg} / 24 \mathrm{~h}$, + vit K). Animals re-

ceived warfarin and vehicle had progressive increase in PT (shown as sINR), whereas co-treatment with vitamin $\mathrm{K}$ prevented sINR increase. Changes in SC levels in control (B), 5/6 nephrectomy rats 3 weeks after the ablative surgery $(5 / 6 \mathrm{NE} 3 \mathrm{~W} ; \mathbf{C}), 5 / 6$ nephrectomy rats 9 weeks after the ablative surgery $(5 / 6 \mathrm{NE} 9 \mathrm{~W}$; D) treated with warfarin $(0.75 \mathrm{mg} / \mathrm{kg} / 24 \mathrm{~h})$ and either vehicle ( $\mathrm{n}=6$ in each group) or vitamin $\mathrm{K}(40 \mathrm{mg} / \mathrm{kg} / 24 \mathrm{~h})\left(\mathrm{n}=6\right.$ in each group). ${ }^{*} \mathrm{p}<0.05$ compared to vehicle-treated animals. Serum creatinine was measured before the surgery and weekly thereafter until treatment with warfarin and vitamin $\mathrm{K}$ began.

ter the cessation of warfarin treatment in $5 / 6 \mathrm{NE}$ rats treated at both $3 \mathrm{~W}$ and $8 \mathrm{~W}$. Animals were monitored until 22 weeks after ablative surgery and SC was measured weekly. There was no difference in the rate of SC increase between non-treated 5/6NE animals and animals who developed AKI associated with warfarin treatment

(WRN) after warfarin treatment was stopped (fig. 5). Morphologically, there was no significant difference in the degree of chronic kidney injury at 22 weeks after ablative surgery between $5 / 6 \mathrm{NE}$ animals treated with warfarin either at $3 \mathrm{~W}$ or $8 \mathrm{~W}$ and vehicle-treated $5 / 6 \mathrm{NE}$ animals. 


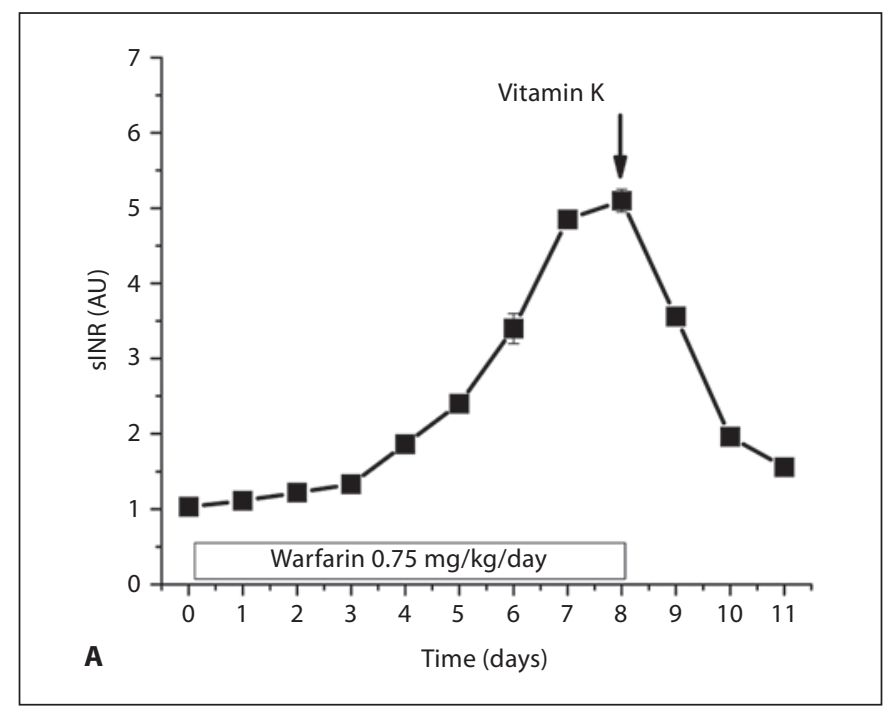

Fig. 5. Changes in serum creatinine (SC) levels in 5/6 nephrectomy rats after a single episode of warfarin-related nephropathy. A 5/6 nephrectomy rats 8 weeks after the ablative surgery were treated with warfarin $(0.75 \mathrm{mg} / \mathrm{kg} / 24 \mathrm{~h})$ for 8 days. This resulted in a rapid prothrombin time (PT) (shown as sINR) increase of more that 4 -fold. Treatment with warfarin was stopped and vitamin $\mathrm{K}(40 \mathrm{mg} / \mathrm{kg})$ was administered. This resulted in a rapid PT decline. B, C SC was increased with PT increase in 5/6 nephrectomy rats treated with warfarin 8 weeks after the ablative surgery (solid circles, $\mathrm{n}=6$; $\mathbf{B}$ ) or 3 weeks after the ablative surgery (solid circles, $n=6 ; \mathbf{C}$ ). The time of the treatment with warfarin is scaled differently; the duration of warfarin treatment is shown as a solid line. After the warfarin treatment cessation and PT normalization, SC levels returned to the levels comparable with 5/6 nephrectomy rats treated with vehicle (no warfarin) 3 and 8 weeks after the ablative surgery (solid squares, $n=9$ ). SC levels were monitored daily in both experimental groups until 22 weeks after the ablative surgery.

\section{Discussion}

To the best of our knowledge, this is the first study demonstrating that WRN may be reproduced in an animal model under the conditions close to those of patients receiving warfarin therapy. WRN is a newly recognized complication of warfarin therapy which we recently characterized based on kidney biopsy [1] and analysis of laboratory data in CKD [2] and non-CKD [3] patients. The current data expand and enhance our previous observations that rapid anticoagulation with brodifacoum (superwarfarin) mimics WRN in 5/6NE [4]. We had earlier demonstrated that 5/6NE animals treated with brodifacoum had elevated SC and morphologic findings in the
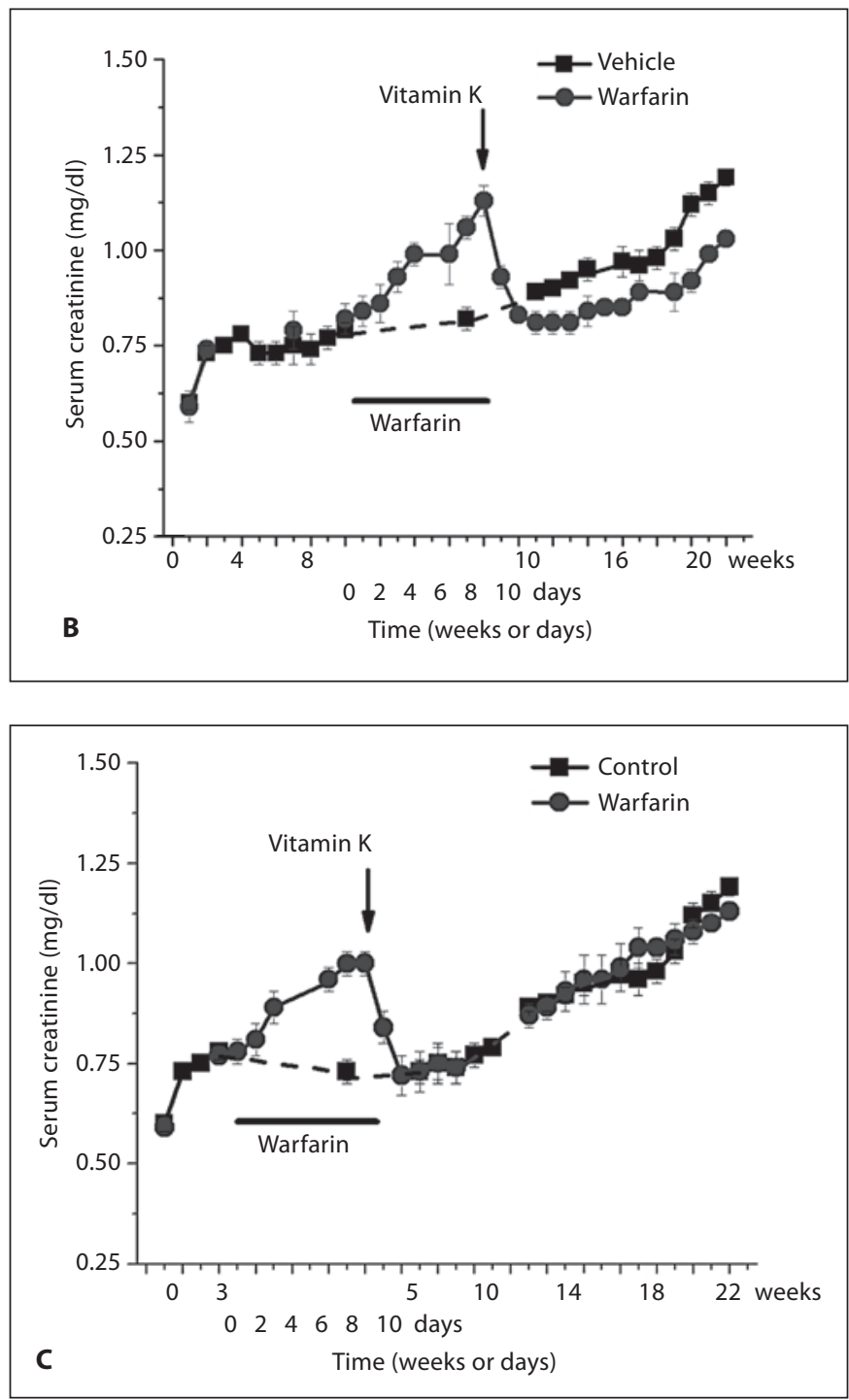

kidneys similar to those observed in patients with WRN [4]. However, treatment with brodifacoum results in a very rapid increase in sINR to $>5$ and the animals did not survive beyond 4 days. These experimental conditions do not mimic a clinical scenario, therefore a need in the studies of effects of anticoagulant therapy on kidney function in the conditions close to the human still existed. In the current work we treated 5/6NE animals with increasing warfarin doses with controlled sINR, which is close to the patients on warfarin therapy. This allowed us to study not only the effects of extremely high sINR on renal function, but 'therapeutic ranges' of sINR as well. The current work provides more evidence that the 5/6NE model is suitable to study the pathogenesis of WRN and 
to develop pharmacologic approaches for WRN treatment and prevention.

Evidence that the warfarin treatment was mechanistically related to its anticoagulation effects includes the following: (1) warfarin affected SC levels in a dose-response manner; (2) the kidney injury included glomerular hemorrhage with occlusive RBC cast formation; (3) with the progression of the $\mathrm{CKD}$, the association between $\mathrm{SC}$ changes and PT was stronger; (4) vitamin K prevented the SC increase and the morphologic changes in the kidneys associated with warfarin treatment, indicating that a coagulopathy is necessary to induce WRN; (5) reversal of the warfarin coagulopathy with vitamin $\mathrm{K}$ results in a return of SC to baseline. This is the first objective evidence that WRN can be treated by warfarin cessation and vitamin $\mathrm{K}$ administration.

Interestingly, the $5 / 6 \mathrm{NE}$ animals that were the most sensitive to PT changes were those treated with warfarin at either 3W or 19W. In these animals SC changes were more strongly correlated with PT changes than in the animals treated with warfarin starting at $8 \mathrm{~W}$. It is difficult to explain these differences based on our findings. We hypothesize that it may be related to the dynamical pathophysiologic changes after the ablative surgery, which include the following phases: (1) the rapid hypertrophic stage (2-4 weeks after ablation), (2) the quiescence stage with minimal histological changes (4-10 weeks after ablation) and (3) the progressive segmental glomerular sclerosis, interstitial fibrosis and tubular atrophy stage (10 weeks and more after ablation) [6-9]. It is possible that during the quiescence phase (4-10 weeks after the ablative surgery) the remaining glomeruli adapt to glomerular hyperfiltration/hyperperfusion injury and they are more resistant to external damaging factors, e.g. injury induced by warfarin or coagulopathy, but this notion will require additional experimental confirmation.

Of note is that we documented RBC and focal RBC casts in 5/6NE animals treated with warfarin even in the 'therapeutic' range of sINR increase (between 2 and 3). This suggests that in humans WRN could occur even though the INR is in the therapeutic range. Although that is plausible, we did not observe this in our studies in humans $[2,3]$. That is, the increase in SC was distinctly noted only in the period one week prior to the INR $>3.0$. Our clinical studies were not prospective. Therefore, we cannot be certain that the INR was above the therapeutic range in the period that immediately preceded the INR >3.0. However, our clinical data are consistent with the notion that WRN does not require a prolonged period with INR above the therapeutic range.
We provide evidence that correction of the warfarin coagulopathy by vitamin K not only prevents WRN, but also promotes recovery from WRN. Also, we show that the progression of CKD was not affected by a single episode of WRN. These findings differ from those of our patients with WRN, where we found that WRN generally accelerates the progression of CKD [3]. This discordance may be explained by the following: (1) only young animals were used for the present studies as opposed to the generally elderly patients with multiple comorbidities in our previous work [1-3]; (2) the degree of chronic kidney injury in experimental animals at the time of WRN development was mild, whereas many patients with CKD had at least moderate chronic kidney injury and decline in renal function; (3) WRN in experimental animals was controlled and the duration of increased SC levels was minimal, as opposed to the patients, whose duration of increased INR is often unknown and who often have no evaluation of renal function until a severe decline in renal function develops.

In conclusion, we provide evidence that the 5/6NE model is a suitable model to study the pathogenesis of WRN that is seen in humans because of the close parallel between the animal model and the human disease. Although our work in the animal model clearly shows the link between the coagulopathy of warfarin and WRN, the cellular and biochemical mechanisms that caused the glomeruli to hemorrhage is not clear. Understanding these mechanisms and whether they are relevant only to the coagulopathy induced by warfarin remains to be established. The present WRN model should be helpful in elucidating these important pathophysiologic questions.

\section{Acknowledgement}

The study was supported in a part by a start-up fund for SVB provided by the Department of Pathology, The Ohio State University.

\section{Disclosure Statement}

None of the authors have any sponsorship or funding arrangements relating to this research to disclose. 


\section{References}

-1 Brodsky SV, Satoskar A, Chen J, Nadasdy G, Eagen JW, Hamirani M, Hebert L, Calomeni E, Nadasdy T: Acute kidney injury during warfarin therapy associated with obstructive tubular red blood cell casts: a report of 9 cases. Am J Kidney Dis 2009;54:1121-1126.

-2 Brodsky SV, Collins M, Park E, Rovin BH, Satoskar AA, Nadasdy G, Wu H, Bhatt U, Nadasdy T, Hebert LA: Warfarin therapy that results in an International Normalization Ratio above the therapeutic range is associated with accelerated progression of chronic kidney disease. Nephron Clin Pract 2010;115:c142-c146.

-3 Brodsky SV, Nadasdy T, Rovin BH, Satoskar AA, Nadasdy GM, Wu HM, Bhatt UY, Hebert LA. Warfarin-related nephropathy occurs in patients with and without chronic kidney disease and is associated with an increased mortality rate. Kidney Int 2011;80: 181-189.
-4 Ware K, Brodsky P, Satoskar AA, Nadasdy T, Nadasdy G, Wu H, Rovin BH, Bhatt U, Von Visger J, Hebert LA, Brodsky SV: Warfarinrelated nephropathy modeled by nephron reduction and excessive anticoagulation. J Am Soc Nephrol 2011;22:1856-1862.

5 Subject: Revision of the Guide for the Care and Use of Laboratory Animals. Bethesda, Md., U.S. Department of Health and Human Services Public Health Services, National Institutes of Health, Publication Number 8623, 1985.

6 Fogo AB: Animal models of FSGS: lessons for pathogenesis and treatment. Semin Nephrol 2003;23:161-171.
7 Brenner BM: Nephron adaptation to renal injury or ablation. Am J Physiol 1985; 249:F324-F337.

-8 Gretz N, Meisinger E, Waldherr R, Strauch M: Acute renal failure after $5 / 6$ nephrectomy: histological and functional changes. Contrib Nephrol 1988;60:56-63.

9 Waldherr R, Gretz N: Natural course of the development of histological lesions after 5/6 nephrectomy. Contrib Nephrol 1988;60:6472.

10 Racusen LC, Solez K, Colvin RB, Bonsib SM, Castro MC, Cavallo T, Croker BP, Demetris AJ, Drachenberg CB, Fogo AB, Furness P, Gaber LW, Gibson IW, Glotz D, Goldberg JC, Grande J, Halloran PF, Hansen HE, Hartley B, Hayry PJ, Hill CM, Hoffman EO, Hunsicker LG, Lindblad AS, Yamaguchi Y, et al: The Banff 97 working classification of renal allograft pathology. Kidney Int 1999;55:713723 